\title{
Evaluar el nivel de satisfacción del cliente de las entidades financieras del municipio de Pamplona
}

\section{Evaluating the customer's satisfaction level the financial entities of the municipality of Pamplona}

\section{Zoraima Victalia Peñaranda Ayala1 ${ }^{1}$;Alfonso Quijano Parra² ,;Saury Thomas Manzano ${ }^{3}$}

${ }^{1}$ Ingeniera Industrial, Especialista en Alta Gerencia.

${ }^{2}$ Grupo de Investigación en Química. Laboratorio de Control de Calidad Universidad de Pamplona-Colombia.

${ }^{3}$ Ingeniero Industrial - Esp. en Estadística Aplicada-Magister Gerencia de Empresas Mención Finanzas. Universidad de Pamplona.

\section{Resumen}

Para las entidades financieras del municipio de pamplona lograr la satisfacción del cliente es un reto diario, sujeto a la calidad del servicio, satisfacción laboral y dar cumplimiento con los requisitos que la entidad le ofrece al cliente. Por lo anterior les permitirá ser más productivos y competitivos toda vez que la competencia entre los servicios es muy alta, es por esto que el objetivo de la presente investigación es evaluar el nivel de satisfacción del cliente de las entidades financieras, bajo el desarrollo de la investigación descriptiva, inferencial y explicativos y cuantitativa y de campo. La población objeto está dada por los clientes permanentes y transitorios y los empleados de las entidades financieras. La muestra calculada para los clientes es de 381 y 91 para los empleados. El instrumento de recolección de información utilizado fue la encuesta el cual fue analizado en el SPSS versión 23 en español aplicando la estadística descriptiva e inferencial. Los resultados obtenidos de la investigación es que no existe una satisfacción laboral, por lo que se recomienda a las entidades financieras del municipio de Pamplona realizar un estudio de teoría de Colas que permita identificar las falencias a la hora de prestar el servicio.

Palabras clave: satisfacción del cliente, calidad del servicio, productividad y satisfacción laboral

\section{Abstract}

For the financial entities of the municipality of pamplona to achieve customer satisfaction is a daily challenge, subject to quality of service, job satisfaction and comply with the requirements that the entity offers the client. Therefore, it will allow them to be more productive and competitive, since competition between services is very high. This is why the objective of this research is to evaluate the level of customer satisfaction of financial institutions under the development of Descriptive, inferential and explanatory and quantitative and field research. The target population is given by permanent and transitory clients and employees of financial institutions. The sample calculated for customers is 381 and 91 for employees. The instrument of information collection used was the survey which was analyzed in SPSS version 23 in Spanish applying the descriptive and inferential statistics. The results obtained from the research is that there is no job satisfaction, so it is recommended to the financial institutions of the municipality of Pamplona to conduct a study of queues theory to identify failures when providing the service.

Keywords: customer satisfaction, quality of service, productivity and job satisfaction 
Hoy en día las empresas luchan por mantenerse y posicionarse en el mercado aumentando los niveles de productividad y competitividad, logrando altos resultados de eficiencia a un menor costo, mediante la incursión y aplicación de innovación y creatividad utilizando mejores métodos de trabajo o prestación de un buen y mejor servicio en cada uno de los procesos relacionados con la actividad a la que se dedica la organización, mediante la incursión de tecnología, selección del personal apto para su desempeño, permitiéndole a si a la organización el aumento y manejo de información enfocada al crecimiento de las empresas, apertura a nuevos mercados, generando credibilidad y fidelizando a sus clientes y que estos se sientan más satisfechos con la calidad del servicio prestado, para Juan Carlos Bustamante (2015), el cliente es el eje central de cualquier organización. Teniendo como referencia que en la actualidad la satisfacción del cliente se ha convertido en un tema fundamental para que las empresas logren alcanzar los objetivos trazados, mantenerse y posicionarse en el mercado aumentando los niveles de productividad y competitividad, logrando altos resultados de eficiencia a un menor costo siendo los clientes quienes le proporcionen a las organizaciones altas utilidades de sostenibilidad, crecimiento e incursión a nuevos mercados es por esto que se debe tener presente que los clientes cada día exigen más por la calidad del producto o servicio en cuanto a seguridad, tiempos de entrega, mercadeo, respeto, cercanía y comunicación. Wang (2003), la alta calidad del servicio prestado define el éxito y supervivencia de la empresa. (Chao 2008) sustenta la calidad del servicio como un antecedente de lealtad del cliente, siendo la lealtad un escalafón que le da a la organización estabilidad económica es decir la permanencia de un cliente es mas económico que la búsqueda de un nuevo cliente, en Colombia las empresas con mayor nivel de satisfacción son las empresa grandes y reconocidas, estas empresas han logrado estos grados de satisfacción empleando estrategias enfocadas a la satisfacción laboral, calidad del servicio y satisfacción laboral. Por lo que se concluye que en la actualidad las empresas no solo les debe preocupar cumplir con las exigencias y requisitos del cliente para su respectiva satisfacción si no que existe una variable de igual importancia y es mantener a sus empleados satisfechos en cuanto a puestos de trabajo que se ajusten a las actividades que desempeña, excelente remuneración, incentivos, capacitaciones, permanencia en los puestos de trabajo y rotación periodica de jefes y lo más importante reconocer la labor que desempeña por la organización, Newstrom, 2007 sostiene que la satisfacción laboral se define como un sentimiento con que los empleados perciben el trabajo, aquel empleado que este insatisfecho con el trabajo o las actividades que desempeña lo reflejara con la respuesta y trato al cliente. Es por esto que el objetivo de la investigación está enmarcada en evaluar el nivel de satisfacción del cliente de las entidades financieras del municipio de pamplona, toda vez que en la actualidad estas entidades financieras del municipio se enfrentan a un alto nivel de competencias por los diferentes servicios que estos prestan llámense créditos, microcréditos transacciones, CDTS, tarjetas débito y crédito entre otros servicios, para Goode y Moutinho (1996) quien argumenta que por la amplia oferta de productos y la disminución de los costos hacen que las entidades financieras tengan un 
alto índice de competencia incitando a los investigadores de Marketing a tener como referente la satisfacción del cliente como factor primordial para conservar la lealtad por la organización. En este orden de ideas un cliente satisfecho es aquel a quien le han suministrado un servicio con calidad y este le proporciona a la entidad lealtad y permanencia, Feria, Herrera y Hernández (2013) sostienen que la calidad del servicio se refleja en el grado de satisfacción del cliente y este hacia la lealtad por la organización, también afirman que las empresas de servicios deben determinar qué beneficios esperan recibir los clientes para que las organizaciones fijen metas y estrategias de cómo satisfacer sus expectativas, por otro lado según Juan Carlos Bustamante (2015) la calidad del servicio se fundamenta en evaluaciones referentes a características intrínsecas al servicio. Por consiguiente, si la empresa tiene claro como brindar un excelente servicio que logre la permanencia de los clientes dentro de la organización dará por hecho la satisfacción del cliente y el mayor grado de competitividad.

Por lo anterior se vio la necesidad de diseñar estrategias que permitan evaluar la calidad del servicio, Ruyteret, 1997; Ghobadian, 1994 definen la calidad como el antecedente de la satisfacción del cliente. Por consiguiente para la investigación se identificaron las necesidades y exigencias de los clientes de las entidades financieras del municipio de pamplona y a su vez evaluar al personal que está a cargo de los procesos para la generación del valor al servicio y así lograr permanencia y lealtad de nuestros clientes, en el municipio de pamplona, para que esto se de se debe tener presente que el cliente es muy exigente y es el que le da el tiempo, crecimiento y posicionamiento en un mercado abierto. Teniendo en cuanta lo anterior el trabajo se fracciona en tres aspectos importantes para la investigación los cuales se definen así:

Aspecto Social: Los principales favorecidos serán la población de pamplona y la totalidad de clientes y usuarios de las entidades bancarias, por la mejora en la calidad del servicio posibilitando cumplir con las expectativas a corto, mediano y largo plazo de sus usuarios y clientes.

Aspecto Económico: En este aspecto los mayores beneficiarios serán las mismas entidades financieras, ya que con la mejora del servicio permite mantener la lealtad de sus clientes y la atracción, a su vez de nuevos clientes con miras a mejorar los costos de operación, manejo y expansión a nuevas sucursales, también permitirle a las organizaciones aumento en los créditos y demás manejos financieros que estos manejan.

Aspecto Metodológico: Incitar a futuros investigadores para la generación de nuevos estudios a este sector u otros similares que aporten nuevas tecnologías, 
97

herramientas o planteamientos que permitan mejorar los servicios y satisfacción de los clientes y usuarios.

\section{MARCO TEORICO}

Una empresa que mantiene un cliente satisfecho es aquella que es productiva y competitiva cuya meta está enfocada en el crecimiento del nicho del mercado y la permanencia de sus clientes, para lo cual existen factores que las organizaciones deben tener presentes para dar cumplimiento en cuanto a satisfacción y lealtad, dentro de estos factores se encuentra la calidad del servicio debe ajustarse a las expectativas y requerimiento de los clientes, ya que los clientes perciben la calidad por el tipo de desempeño, la forma de ejecución del servicio, veracidad y exactitud e innovación, precios, métodos de trabajo que este utiliza, tipos de comunicación e información que las organizaciones tienen con el cliente, Iguarán, Chávez y Pérez (2006) confirman que la información que recibe el cliente por parte de la organización puede modificar considerablemente su percepción de la calidad del servicio, para Romero y Romero (2006), sustenta la necesidad de que existan nuevos canales de comunicación con el cliente que le permitan mantenerse informado y que este pueda percibir adecuadamente las dimensiones del servicio ofrecido. Por otro lado Ko y Pastore (2005) confirman que la información es un elemento que se debe utilizar en el entorno de la organización para la utilización en el discernimiento de la calidad del servicio. En este orden de ideas que la organización esté cumpliendo con sus expectativas y requisitos esperados que le brinde la organización al cliente, Pizzo (2013) describe la calidad del servicio como un habito que la organización ha desarrollado y practicado para interpretar las necesidades y expectativas de sus clientes y ofrecerles, en consecuencia, un servicio accesible, adecuado, ágil, flexible, apreciable, útil, oportuno, seguro y confiable, incluso bajo circunstancias inesperadas, de tal manera que el cliente se sienta comprendido, atendido y servido personalmente.

Por lo anterior es de vital importancia tanto para los investigadores como para las organizaciones el tema de calidad de servicio en cuanto a la satisfacción del cliente y el manejo adecuado de los factores o elementos que hacer que una organización de la calidad que el cliente necesita enfocada a la comunicación, información exactitud y pronta respuesta de los servicios.

Para Peralta (2004) la calidad del servicio ha ido en aumento y se le ha dado el reconocimiento al talento humano, aumentando la satisfacción laboral que se refleje en un excelente servicio que le permita a la organización ser más 
productivo y competitivo, es por ello que la satisfacción laboral es un determinante económico, de carácter eficiente y competitivo para la organización que la puede llevar a ser incompetente y no productivo, según la revista Artices en el 2011, en su artículo Satisfacción Laboral=Productividad, en donde señala el autor que han sido muchas las empresas que han descuidado a sus empleados y que esto se identifica al medir el clima organizacional, en donde esté presente baja productividad en el comportamiento organizacional, bajo sentido de pertenencia por el trabajo a desempeñar y actitud desdeñosa para el desempeño laboral por lo que se concluye que un empleado satisfecho refleja sus emociones o estados de ánimo al cliente generándole a la organización un servicio con calidad. Por su parte Guru, 2003 manifiesta que la calidad del servicio ha tenido un impacto en el desempeño de los negocios ya que ha generado reducción en costos lealtad de los clientes y rentabilidad, lo que lleva a las organizaciones al aumento del nicho del mercado y al posicionamiento en cuanto a marketing.

A su vez la definición de satisfacción del cliente está dada por el grado de conformidad, confianza, compromiso reputación, expectativas y lealtad que haya adquirido o tenga el cliente del servicio recibido o producto comprado con relación a la calidad, con referencia a lo anterior para (Setó 2004) la satisfacción del cliente depende de la calidad del servicio, en otras palabras si el servicio es transparente y cumple con las expectativas y requerimientos que espera el cliente, este le generara confianza y esta a su vez le proporcionara a las organizaciones lealtad y permanencia.

En efecto para Zeithaml y Bitner (2002), la satisfacción del cliente es la valoración que tiene el cliente con relación al producto o servicio y a su vez si este cumple con todas las expectativas y la totalidad de la necesidad del servicio. Los autores en la teoría enuncian cinco patrones en la calidad de la satisfacción. De acuerdo con Baptista y León (2013), para los servicios la satisfacción se muestra cuando el cliente ve reflejado la calidad en todas las dimensiones que conforman el servicio o producto y que a su vez que cumplan con todas las expectativas y requerimientos que el cliente espera, por consiguiente, los autores destacan cinco patrones: Tangibilidad, Empatía, Confiabilidad, Seguridad y Capacidad de Respuesta.

Dados los criterios o elementos existentes como soporte teórico para la investigación que permitieron el desarrollo del objetivo de estudio de evaluar el nivel de satisfacción del cliente las entidades financieras del municipio de pamplona

\section{METODOLOGIA}


99

La presente investigación está enfocada en evaluar el nivel de satisfacción del cliente en las entidades financieras de la ciudad de Pamplona y a su vez la calidad del servicio que estos prestan mediante la aplicación de la investigación descriptiva, inferencial, explicativa y cuantitativa e investigación de campo, dando al tipo de investigación e información y datos de forma directa y real. Para la investigación explicativa según Hernández, Fernández y Collado (2010), "los estudios explicativos van más allá de la descripción de conceptos o fenómenos o del establecimiento de relaciones entre conceptos; es decir, está dirigido a responder las causas de los eventos y fenómenos físicos o sociales. Para el fortalecimiento del objeto de estudio se identificó los tipos de población, Según Martínez (2012) la población o universo es un conjunto de unidades o elementos que presentan una característica común, también se le considera como un conjunto de medias. Para la presente investigación la población está dada por todas las personas que están influenciadas con las entidades financieras del municipio de pamplona (Clientes o usuarios y Empleados). La investigación se dividió en dos poblaciones a quienes se les aplico una serie de preguntas dentro del marco de encuesta, con la finalidad de obtener mayor exactitud en los resultaos y así dar el cumplimiento de los objetivos que nos permitan llegar al diseño o sugerencias de estrategias que permitan aumentar el nivel de satisfacción de los clientes y usuarios de las entidades financieras de la ciudad de Pamplona. Las poblaciones son:

Población de estudio 1: Conformada por los Clientes y usuarios permanentes y transitorios de las entidades financieras de la ciudad de Pamplona

\begin{tabular}{|l|l|}
\hline Banco O Cooperativa & $\mathrm{N}^{\circ}$ de Clientes \\
\hline BANCOLOMBIA & 3.980 \\
\hline BANCO DE BOGOTA & 8.150 \\
\hline BBVA & 5.020 \\
\hline BANCOOMEVA & 3.100 \\
\hline BANCO POPULAR & 5.100 \\
\hline DAVIVIENDA & 4.010 \\
\hline BANCO AGRARIO & 4.955 \\
\hline BANCAMIA & 3.510 \\
\hline COOMULTRASAN & 6.350 \\
\hline BANCO DE LA MUJER & 4.010 \\
\hline CREZCAMOS & 993 \\
\hline
\end{tabular}


Fuente: Personal a cargo de los procesos de cada entidad.

Población de estudio 2: Todos los trabajadores directos y aquellos que interactúen con cada proceso de las entidades financieras de la ciudad de Pamplona

\begin{tabular}{|c|c|c|c|c|}
\hline \multicolumn{5}{|c|}{ EMPLEADOS POR ENTIDAD FINANCIERA } \\
\hline $\begin{array}{l}\text { BANCO O } \\
\text { COOPERATIVA }\end{array}$ & $\begin{array}{l}\text { NÚMERO DE } \\
\text { EMPLEADOS }\end{array}$ & $\begin{array}{l}\text { BANCO O } \\
\text { COOPERATIVA }\end{array}$ & $\begin{array}{l}\text { NÚMERO } \\
\text { EMPLEADOS }\end{array}$ & $\mathrm{DE}$ \\
\hline BANCOLOMBIA & 13 & DAVIVIENDA & 9 & \\
\hline $\begin{array}{ll}\text { BANCO } & \text { DE } \\
\text { BOGOTA } & \\
\end{array}$ & 15 & $\begin{array}{l}\text { BANCO } \\
\text { AGRARIO }\end{array}$ & 10 & \\
\hline BBVA & 13 & BANCAMIA & 10 & \\
\hline BANCOOMEVA & 7 & $\begin{array}{l}\text { COOMULTRASA } \\
\mathrm{N}\end{array}$ & 11 & \\
\hline $\begin{array}{l}\text { BANCO } \\
\text { POPULAR }\end{array}$ & 10 & $\begin{array}{lll}\text { BANCO DE } & \text { LA } \\
\text { MUJER } & & \end{array}$ & 13 & \\
\hline CREZCAMOS & 9 & & & \\
\hline TOTAL & & & 120 & \\
\hline
\end{tabular}

Fuente: Personal a cargo de los procesos de cada entidad.

Para determinar la muestra de las poblaciones uno y dos se aplicó la siguiente ecuación:

$$
\begin{aligned}
& n=\frac{p \times q}{\frac{e^{2}}{Z^{2}}+\frac{p \times q}{N}} \quad \mathbf{Z}=\text { Grado de confianza. Para esta investigación, se aplicó una confiabilidad } \\
& \text { del 95\% que equivale a un valor de } \mathrm{Z} \text { de } 1.96 \text {. } \\
& \mathbf{e}=\text { Margen de error. Para este proyecto el margen utilizado es del 4,5\%. }
\end{aligned}
$$

$\mathbf{N}=$ Tamaño de la población, para la población uno y dos se aplicó el muestreo aleatorio simple estratificado.

$\mathbf{p}=$ probabilidad de éxito, será igual 0,5 , esto debido a que no se realizó una prueba piloto.

$\mathbf{q}=$ probabilidad de fracaso, será igual 0,5 , esto debido a que no se realizó una prueba piloto.

\section{RESULTADOS}

Se realizó el análisis de los resultados obtenidos aplicados a las dos poblaciones descritas en la metodología, para este análisis se utilizó el software SPSS versión 23 en español con la finalidad de analizar y aplicar la estadística descriptiva e inferencial en la investigación el cual le da mayor precisión al análisis de los resultados para que la investigación sea más robusta.

\section{ANALISIS DELOS EMPLEADOS O ADMINISTRATIVOS DE LAS ENTIDADES FINANCIERAS DEL MUNICIPIO DE PAMPLONA}

Obtenida la muestra (91) se determinó el muestro aleatorio simple, este análisis permite demostrar que la muestra obtenida a través de la fórmula del muestreo es exacta. 


\begin{tabular}{|l|l|l|l|}
\hline $\begin{array}{l}\text { BANCO O } \\
\text { COOPERATIVA }\end{array}$ & $\begin{array}{l}N^{\circ} \text { DE } \\
\text { EMPLEADOS }\end{array}$ & PESO RELATIVO & MUESTRA \\
\hline BANCOLOMBIA & 13 & 0,108 & 10 \\
\hline $\begin{array}{l}\text { BANCO DE } \\
\text { BOGOTA }\end{array}$ & 15 & 0,125 & 11 \\
\hline BBVA & 13 & 0,108 & 10 \\
\hline BANCOOMEVA & 7 & 0,058 & 5 \\
\hline BANCO POPULAR & 10 & 0,083 & 8 \\
\hline DAVIVIENDA & 9 & 0,075 & 7 \\
\hline BANCO AGRARIO & 10 & 0,083 & 7 \\
\hline BANCAMIA & 10 & 0,083 & 8 \\
\hline COOMULTRASAN & 11 & 0,092 & 8 \\
\hline $\begin{array}{l}\text { BANCO DE LA } \\
\text { MUJER }\end{array}$ & 13 & 0,108 & 10 \\
\hline CREZCAMOS & 9 & 0,075 & 7 \\
\hline TOTAL & 120 & 1,000 & 91 \\
\hline
\end{tabular}

Como se puede ver en la tabla anterior del Muestreo Aleatorio Simple se evidencia y comprueba que la muestra da igual a la hallada por la ecuación y el peso relativo da un valor igual a uno (1). Por lo anterior aplicado el instrumento de recolección de información se procesó y analizado en el software SPSS versión 23 en español, el análisis está representado mediante análisis descriptivo e inferencial y tablas de contingencia, en donde se analizaron las preguntas de mayor relación que permitieron abrir el horizonte para lograr obtener el grado de satisfacción laboral que se ve reflejado en la calidad del servicio y la satisfacción de los clientes de las entidades financieras del municipio de Pamplona.

Una de las preguntas de mayor relevancia dentro del análisis descriptivo estadístico es el cruce entre las preguntas 4 Número de Clientes o Usuarios que atienden diariamente con la Pregunta : 5 Tiempo promedio que demora en atender un cliente o usuario. En la cual se obtuvo la siguiente información.

\begin{tabular}{|c|c|c|c|c|c|c|c|}
\hline \multicolumn{8}{|c|}{$\begin{array}{l}\text { Tabla cruzada Pregunta : 4. Número de Clientes o Usuarios que atienden diariamente } * \text { Pregunta : 5. Tiempo } \\
\text { promedio que demora en atender un cliente o usuario }\end{array}$} \\
\hline & & \multicolumn{5}{|c|}{$\begin{array}{r}\text { Pregunta : } 5 \text { Tiempo promedio que demora en atender un } \\
\text { cliente o usuario }\end{array}$} & \multirow{2}{*}{ Total } \\
\hline & & \begin{tabular}{|r|} 
Menos de \\
2 minutos \\
\end{tabular} & $\begin{array}{r}\begin{array}{r}\text { Entre 2 } \\
\text { minutos }\end{array} \\
\end{array}$ & $\begin{array}{r}\begin{array}{r}\text { Entre } 4 \text { y } 6 \\
\text { minutos }\end{array} \\
\end{array}$ & $\begin{array}{r}\begin{array}{r}\text { Entre } 6 \text { y } 8 \\
\text { minutos }\end{array} \\
\end{array}$ & $\begin{array}{r}\begin{array}{r}\text { Mayor a } 8 \\
\text { minutos }\end{array} \\
\end{array}$ & \\
\hline \multirow{5}{*}{$\begin{array}{l}\text { Pregunta : } 4 \text { Número } \\
\text { de Clientes } \\
\text { Usuarios que } \\
\text { atienden diariamente }\end{array}$} & \begin{tabular}{|lll}
$\begin{array}{l}\text { Menor de } \\
\text { Clientes }\end{array}$ & 10 \\
\end{tabular} & 1 & 0 & 1 & 0 & 0 & \\
\hline & 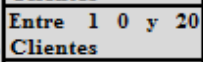 & 0 & 0 & 0 & 6 & 1 & \\
\hline & \begin{tabular}{|l|l} 
Entre 20 y 30 \\
Clientes
\end{tabular} & 2 & 1 & 4 & 24 & 4 & 35 \\
\hline & $\begin{array}{l}\text { Entre } 30 \text { y } 40 \\
\text { Clientes }\end{array}$ & 0 & 2 & 10 & 19 & 4 & 36 \\
\hline & $\begin{array}{l}\text { Mayor a } 40 \\
\text { Clientes }\end{array}$ & 2 & 5 & 3 & 1 & 0 & 11 \\
\hline \multicolumn{2}{|l|}{ Total } & 5 & 8 & 18 & 50 & 9 & 91 \\
\hline
\end{tabular}


Para la tabla el 55\% (50 empleados) representa el tiempo en el que un servidor demora en atender un usuario o cliente, cuyo tiempo oscila entre 6 a 8 minutos, mientras que el otro 19,8\% (18 empleados) se demora entre 4 y 6 minutos, estos dos tiempos están muy altos con relación a la cantidad de clientes y servicios que prestan las entidades financieras, así mismo encontramos que entre 20 y 30 clientes son tendidos en un intervalo de tiempo de 6 a 8 minutos, lo que equivale a el 26,4\% (24 empleados) de atención de cliente. Por lo anterior se denota que se debe implementar una estrategia o plan que permita disminuir los tiempos de atención por parte de los funcionarios de las entidades financieras que les permita agilizar los procesos que satisfagan las necesidades de los clientes.

\section{ANALISIS DESCRIPTIVO DE LOS EMPLEADOS DE LAS ENTIDADES FINANCIERAS DEL MUNICIPIO DE PAMPLONA}

La siguiente tabla de análisis descriptivo fue de vital importancia para la investigación ya que permitió observar, revisar o inferir si existe o no variabilidad en la información suministrad por los empleados de las entidades financieras del municipio de Pamplona 


\begin{tabular}{|c|c|c|c|c|c|c|c|c|c|}
\hline & & $\begin{array}{l}\text { Pregunta } \\
: 1 \text { Nombre } \\
\text { de Ia la } \\
\text { entidad }\end{array}$ & $\begin{array}{l}\text { Pregunta: } 2 \\
\text { Cargo qu } \\
\text { Ocupa en } \\
\text { Entidad }\end{array}$ & $\begin{array}{l}\text { Pregunta: } 3 \\
\text { Años } \\
\text { experiencia } \\
\text { dentro de } \\
\text { entidad } \\
\end{array}$ & $\begin{array}{l} \\
\text { Pregunta: } 4 \\
\text { Número } \\
\text { de Clientes } \\
\text { Usuarios } \\
\text { Ia atienden } \\
\text { diariamente }\end{array}$ & de & $\begin{array}{l}\text { Pregunta = } \\
\text { Tiempo } \\
\text { promedio que } \\
\text { demora } \\
\text { atender un } \\
\text { cliente } \\
\text { usuario }\end{array}$ & $\begin{array}{l}\text { Pregunta: } 6 \\
\text { Considera } \\
\text { Costed que o } \\
\text { aumentarse } \\
\text { nummero } \\
\text { servidores } \\
\text { canales }\end{array}$ & 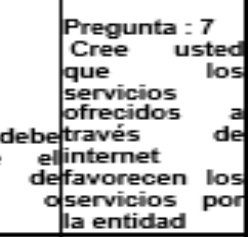 \\
\hline \multirow[t]{2}{*}{$\bar{N}$} & Válido & 91 & 91 & 91 & e1 & & 91 & 91 & 91 \\
\hline & $\begin{array}{l}\text { Perdid } \\
\text { os }\end{array}$ & 0 & 0 & 0 & b & & 0 & 0 & b \\
\hline \multirow{3}{*}{\multicolumn{2}{|c|}{$\begin{array}{l}\text { Media } \\
\text { Error estándar de la } \\
\text { media } \\
\text { Mediana }\end{array}$}} & 5,12 & 3,88 & 2,74 & 3.52 & & 3,58 & 1,13 & 1,88 \\
\hline & & .332 & .207 & .102 & 083 & & .100 & .036 & 034 \\
\hline & & 5,00 & $4, \infty$ & 3,00 & 4,00 & & 4,00 & $1, \infty 0$ & 2,00 \\
\hline \multicolumn{2}{|l|}{ Moda } & $1^{2}$ & 2 & 3 & 4 & & 4 & 1 & 2 \\
\hline \multirow{2}{*}{\multicolumn{2}{|c|}{$\begin{array}{l}\text { Desviación estándar } \\
\text { Varianza }\end{array}$}} & 3,169 & 1.972 & .978 & .888 & & 1.012 & .340 & 328 \\
\hline & & 10,041 & 3,888 & .952 & 780 & & 1,024 & .116 & 107 \\
\hline \multicolumn{2}{|c|}{ Asimetría } & .282 & .431 & -.178 & -344 & & -855 & 2.213 & $-2,365$ \\
\hline \multirow{2}{*}{\multicolumn{2}{|c|}{$\begin{array}{l}\text { Error estándar d } \\
\text { asimetria } \\
\text { Curtosis }\end{array}$}} & .253 & .253 & .253 & 253 & & .253 & .253 & 253 \\
\hline & & $-1,224$ & --.857 & -.685 & 262 & & .828 & 2.961 & 3.674 \\
\hline \multirow{2}{*}{\multicolumn{2}{|c|}{$\begin{array}{l}\text { Error estándar de } \\
\text { curtosis } \\
\text { Rango }\end{array}$}} & .500 & .500 & .500 & .500 & & .500 & .500 & .500 \\
\hline & & 10 & 7 & 4 & 4 & & 5 & 1 & 1 \\
\hline \multicolumn{2}{|l|}{ Mínimo } & 1 & 1 & 1 & 1 & & 1 & 1 & 1 \\
\hline \multirow{2}{*}{\multicolumn{2}{|c|}{$\begin{array}{l}\text { Máximo } \\
\text { Suma }\end{array}$}} & 11 & 8 & 5 & 5 & & 6 & 2 & 2 \\
\hline & & 468 & 362 & 249 & 320 & & 328 & 103 & 171 \\
\hline \multirow[t]{3}{*}{ Percentiles } & 25 & 2,00 & 2,00 & 2,00 & 3,00 & & 3,00 & $1, \infty$ & 2,00 \\
\hline & 50 & 5,00 & 4,00 & 3,00 & 4.00 & & 4,00 & $1, \infty 0$ & 2,00 \\
\hline & 75 & 8.00 & 5,00 & 3,00 & 4.00 & & 4,00 & 1,00 & 2,00 \\
\hline
\end{tabular}

EL análisis descriptivo que se observa anteriormente de los empleados de las entidades financieras del municipio de Pamplona en la que se identificaron aquellas preguntas de mayor relación e importancia para analizar con respecto al objeto de estudio son:

Con relación a la pregunta tres (Años de experiencia dentro de la entidad) el análisis se enfoca en media calculada dando como valor de 2.74, esto quiere decir que en cuanto a experiencia de los empleados en la entidad la mayoría de estos se encuentra entre 6 y 8 años, para la investigación este resultado o factor es de vital importancia ya que se determina que en las entidades no existe cambio o rotación de personal periódicamente lo que le permite a las entidades mantener una satisfacción laboral que se refleja en el desempeño de cada una de las actividades relacionadas con la prestación del servicio que conlleva al logro de satisfacción de los clientes dentro de las entidades financieras del municipio, esta es una estrategia que las empresas manejan hoy día, en cuanto a calidad de servicio, costos de entrenamiento de personal y credibilidad y seguridad para los clientes. Para la misma pregunta se analizó la desviación estándar obtenida cuyo resultado fue de 0.976 , significando que existe cierta variabilidad en los datos, lo que indican que la muestra no es tan homogénea.

Ahora bien, respecto a la pregunta cinco (Tiempo promedio que demora en atender un cliente o usuario) la media es de 3.58, determinando que el tiempo que demora un empleado en atender un cliente oscila entre 6 a 8 minutos, lo que quiere decir que el tiempo utilizado para la prestación del servicio es muy alto lo que infiere que a esta variable debe prestársele una atención especial por parte de la gerencia con el fin de disminuir los tiempos de atención al cliente y así evitar una insatisfacción, así mismo se calculó y analizo la desviación estándar en consideración dando como resultado de 1.012, cuyo rango identifica de 5 ( max 6 , min 1), lo que quiere decir que la desviación es alta y los datos están concentrados en el intervalo de 6 a 8 min, por lo tanto se deben tomar correctivos dentro de las entidades financieras, este dato es relevante para la investigación siendo esta una de las justificaciones importantes que permitió continuar con un estudio riguroso y minucioso para la aplicación de teoría de colas. 
En cuanto al análisis de la pregunta seis (Considera usted que debe aumentarse el número de servidores o canales), la mayoría de los administrativos consideran que no es necesario aplicar o crear un nuevo canal de servicio, esta respuesta no tiene relación con el tiempo de demora en la entrega del servicio por parte de los empleados, analizado lo anteriormente y siendo el tiempo de atención al cliente muy alto se hace necesario incrementar uno o más de dos nuevo canal de servicio y así poder obrar con eficiencia y eficacia como lo exige y requiere el cliente.

Para la pregunta número siete (Cree usted que los servicios ofrecidos a través de internet favorecen los servicios por la entidad), los empleados de las entidades financieras consideran que sí que se hace necesario implementar o fortalecer los canales de servicio vía internet, permitiendo así descongestionar los canales de servicio personal dentro de las sucursales. Para la pregunta en consideración se analizó la desviación estándar con un valor obtenido de 0.328 , como se puede observar este valor dio muy cercano a cero siendo esta variable de comportamiento homogéneo sin variabilidad.

\begin{tabular}{|c|c|c|c|c|c|c|c|c|c|}
\hline \multirow[b]{2}{*}{ Ecuación } & \multicolumn{5}{|c|}{ Resumen del modelo } & \multicolumn{4}{|c|}{ Estimaciones de parámetro } \\
\hline & R cuadrado & $F$ & gl1 & $\mathrm{g} \mid 2$ & Sig. & Constante & b1 & b2 & b3 \\
\hline Lineal & 142 & 14,718 & 1 & 89 &, 000 & 925 &, 040 & & \\
\hline Logarítmico &, 096 & 9,503 & 1 & 89 &, 003 &, 943 &, 136 & & \\
\hline Inverso &, 061 & 5,777 & 1 & 89 &, 018 & 1,229 &,- 281 & & \\
\hline Cuadrático & .228 & 12,994 & 2 & 88 &, 000 & 1,176 &,- 093 &, 012 & \\
\hline Cúbico & 356 & 16,028 & 3 & 87 &, 000 & 684 & 344 &,- 080 &, 005 \\
\hline Compuesto &, 142 & 14,718 & 1 & 89 &, 000 &, 949 & 1,028 & & \\
\hline Potencia &, 096 & 9,503 & 1 & 89 &, 003 &, 961 & 095 & & \\
\hline$s$ &, 061 & 5,777 & 1 & 89 &, 018 &, 158 &,- 195 & & \\
\hline Crecimiento &, 142 & 14,718 & 1 & 89 &, 000 &,- 052 &, 028 & & \\
\hline Exponencial &, 142 & 14,718 & 1 & 89 &, 000 &, 949 &, 028 & & \\
\hline Logística &, 142 & 14,718 & 1 & 89 &, 000 & 1,054 & 972 & & \\
\hline
\end{tabular}

La variable independiente es Pregunta :1 Nombre de la entidad.

En la tabla anterior se observa el $\mathrm{R}^{2}$ con respecto a las regresiones es de 0,356 que corresponde a la regresion cubica y se encuentra dentro del intervalo de $0,3 \leq \mathrm{R}^{2}<0,6$ siendo esta una correlación mínima según INFORMESE (2015) en su Manual de Capacitación de IBM SPSS. Tambien se identifica que la regresion lineal presente un $\mathrm{R}^{2}$ equivalente a 0,142 lo cual nos indica que no existe correlacion entre los datos. Con relacion a los modelos de las regresiones observamos que el modelo a estimar de la regresion lineal esta dado por $\bar{y}=b x+c$, lo que nos indica que su modelo a pronosticar según la tabla es $\bar{y}=0,040 x+0,925$. Con respecto al $F$ calculado de la regresión cubica $(16,028)$ comparada con la F observada con 3 y 87 grados de libertad da un guarismo o valor de 2.71; esto quiere decir que se presentan diferencias significativas en la parte experimental que se está llevando a cabo ya que el $\mathrm{F}$ calculado debe ser $\leq \mathrm{al}$ observado. Con respecto al grado de significancia se observa que ninguno de los valores de las regresiones Lineal, Logarítmico Inverso, Cuadrático, Cúbico, Compuesto, Potencia, S, Crecimiento, Exponencial, Logística no arrojan un 
valor $\leq$ a 0,05 lo que indica cómo se menciona anteriormente que existen diferencia significativas en la fase experimental del proyecto lo que indica se deben tomar estrategias de mejora que permitan aumentar la satisfacción de los clientes de las entidades financieras.

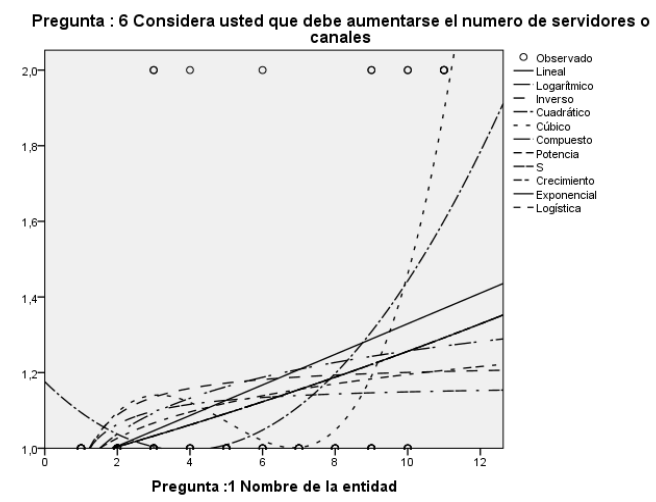

Con relación a la gráfica se observa que no existe un comportamiento de las regresiones: Lineal, Logarítmico Inverso, Cuadrático, Cúbico, Compuesto, Potencia, S, Crecimiento, Exponencial, Logística, esto es debido a que su correlación es mínima, ya que se encuentre en el intervalo de $0,3 \leq$ $\mathrm{R}^{2}<0,6$.

\section{ANÁLISIS DESCRIPTIVO DE LOS CLIENTES DE LAS ENTIDADES FINANCIERAS DEL MUNICIPIO DE PAMPLONA}

\begin{tabular}{|l|r|r|r|}
\hline $\begin{array}{l}\text { BANCO O } \\
\text { COOPERATIVA }\end{array}$ & $\begin{array}{c}\text { No DE } \\
\text { CLIENTES }\end{array}$ & $\begin{array}{r}\text { PESO } \\
\text { RELATIVO }\end{array}$ & MUESTRA \\
\hline BANCOLOMBIA & 3.980 & 0,081 & 31 \\
\hline $\begin{array}{l}\text { BANCO DE } \\
\text { BOGOTA }\end{array}$ & 8.150 & 0,166 & 63 \\
\hline BBVA & 5.020 & 0,102 & 39 \\
\hline BANCOOMEVA & 3.100 & 0,063 & 24 \\
\hline BANCO POPULAR & 5.100 & 0,104 & 40 \\
\hline DAVIVIENDA & 4.010 & 0,082 & 31 \\
\hline BANCO AGRARIO & 4.955 & 0,101 & 38 \\
\hline BANCAMIA & 3.510 & 0,071 & 27 \\
\hline COOMULTRASAN & 6.350 & 0,129 & 49 \\
\hline $\begin{array}{l}\text { BANCO DE LA } \\
\text { MUJER }\end{array}$ & 4.010 & 0,082 & 31 \\
\hline CREZCAMOS & 993 & 0,020 & 8 \\
\hline TOTAL & $\mathbf{4 9 . 1 7 8}$ & $\mathbf{1 , 0 0 0}$ & $\mathbf{3 8 1}$ \\
\hline
\end{tabular}

En la anterior tabla de análisis descriptivo aplicado a los clientes (49178) de las entidades financieras del municipio de Pamplona, se idéntico que existe variabilidad en la información suministrada por los clientes de las entidades financieras del municipio de pamplona ya que el peso relativo hallado dio igual a uno (1) y la muestra es igual a 381.

Permitiéndonos así obtener un análisis de resultados mucho más robusto, preciso en cuanto a segmentación o variables de la investigación y la identificación del grado de satisfacción en cuanto a la prestación del servicio.

Análisis de tablas de contingencia de los clientes de las entidades financieras del municipio de Pamplona: 
Este análisis se aplicó para determinar el grado de relación entre las preguntas aplicadas en el cuestionario a los clientes de las entidades Financieras del Municipio de Pamplona con la finalidad de determinar el grado de satisfacción en cuanto a la calidad del servicio recibido.

\begin{tabular}{|c|c|c|c|c|c|c|c|}
\hline & & \multicolumn{5}{|c|}{$\begin{array}{l}\text { Pregunta : } 6 \\
\text { Cuanto tiempo ha esperado en la cola para ser atendió por } \\
\text { los funcionarios }\end{array}$} & \multirow{2}{*}{ Total } \\
\hline & & $\begin{array}{l}\text { Menor a dos } \\
\text { minutos }\end{array}$ & $\begin{array}{l}\text { Entre } 2 \text { y } 4 \\
\text { min }\end{array}$ & $\begin{array}{c}\text { Entre } 4 \text { y } 6 \\
\min \end{array}$ & $\begin{array}{l}\text { Entre } \\
\text { min }\end{array}$ & $\begin{array}{l}\text { Mayor a } 8 \\
\text { min }\end{array}$ & \\
\hline \multirow{11}{*}{$\begin{array}{l}\text { Pregunta : } 1 \\
\text { De que entidad } \\
\text { financiera es } \\
\text { cliente }\end{array}$} & Bancolombia & 4 & 18 & 2 & 1 & 6 & 31 \\
\hline & Banco de Bogotá & 4 & 4 & 1 & 27 & 26 & 62 \\
\hline & BBVA & 0 & 1 & 2 & 18 & 18 & 39 \\
\hline & Bancomeva & 1 & 1 & 1 & 11 & 10 & 24 \\
\hline & Banco Popular & 1 & 2 & 3 & 19 & 17 & 42 \\
\hline & Davivienda & 5 & 6 & 2 & 4 & 15 & 32 \\
\hline & Banco Agrario & 6 & 6 & 2 & 3 & 15 & 32 \\
\hline & Bancamia & 2 & 3 & 3 & 6 & 13 & 27 \\
\hline & Coomultrasan & 6 & 7 & 3 & 6 & 19 & 41 \\
\hline & \begin{tabular}{|l|} 
Banco de la \\
Mujer
\end{tabular} & 9 & 9 & 2 & 4 & 18 & 42 \\
\hline & Crezcamos & 2 & 3 & of & 0 & 4 & $\mathbf{9}$ \\
\hline \multicolumn{2}{|l|}{ Total } & 40 & 60 & 21 & 99 & 161 & 381 \\
\hline
\end{tabular}

En la tabla anterior se identificó que el 68.2\% (260 clientes) de los clientes han esperado ser atendidos por un servidor o funcionario de las entidades financieras en un intervalo comprendidos entre 6 y 8 minutos y mayor 8 minutos, con lo que se concluye que los tiempos de espera en cola son muy altos y se deben tomar correctivos para agilizar los tiempos de esperas de los clientes o en su defecto instalar más servidores. También se observa que el mayor tiempo de espera se localizó en el banco Bogotá con un 13.9\% (53 clientes) en esperar por ser atendido (mayor igual a 6 minutos intervalos comprendidos entre 6 y 8 minutos y mayor 8 minutos).

\begin{tabular}{|c|c|c|c|c|c|c|c|}
\hline & & \multicolumn{5}{|c|}{$\begin{array}{l}\text { Pregunta } .9 \\
\text { Esta de acuerdo que en horas pico la entidad financiera habilite } \\
\text { un servicio adicional para descongestionar la cola de espera }\end{array}$} & \multirow[t]{2}{*}{ Total } \\
\hline & & \begin{tabular}{|c|} 
Totalmente \\
en \\
Desauerdo
\end{tabular} & \begin{tabular}{|c|} 
En \\
Desacuerdo
\end{tabular} & \begin{tabular}{|c|} 
Le es \\
Indiferente
\end{tabular} & De Acuerdo & $\begin{array}{l}\text { Totalmente } \\
\text { de Acuerdo }\end{array}$ & \\
\hline \multirow[t]{5}{*}{ Pregunta: 1} & Bancolombia & 4 & 3 & 16 & 8 & 0 & 31 \\
\hline & \begin{tabular}{|l} 
Banco de \\
Bogotá \\
\end{tabular} & 6 & 5 & 32 & 17 & 2 & 62 \\
\hline & BBVA & 1 & 3 & 21 & 10 & 4 & 39 \\
\hline & Bancomeva & 0 & 2 & 13 & 8 & 1 & 24 \\
\hline & \begin{tabular}{|l|} 
Banco \\
Popular \\
\end{tabular} & 0 & 1 & 1 & 40 & 0 & 42 \\
\hline \multirow{6}{*}{$\begin{array}{c}\text { De que } \\
\text { entidad } \\
\text { financiera } \\
\text { es cliente }\end{array}$} & Davivienda & 0 & 0 & 1 & 29 & 2 & 32 \\
\hline & \begin{tabular}{|l|} 
Banco \\
Agrario \\
\end{tabular} & 0 & 0 & 3 & 14 & 15 & 32 \\
\hline & Bancamia & 1 & 0 & 7 & 18 & 1 & 27 \\
\hline & \begin{tabular}{|l|} 
Coomultrasa \\
n \\
\end{tabular} & 1 & 1 & 13 & 6 & 20 & 41 \\
\hline & $\begin{array}{l}\text { Banco de la } \\
\text { Mujer } \\
\end{array}$ & 2 & 0 & 10 & 11 & 19 & 42 \\
\hline & Crezcamos & 0 & 0 & 0 & 5 & 4 & 9 \\
\hline \multicolumn{2}{|l|}{ Total } & 15 & 15 & 117 & 166 & 68 & 381 \\
\hline
\end{tabular}

El 61.4\% (234 clientes ) de los clientes respondió que está de acuerdo que en horas pico se habilite un servicio adicional que agilice el servicio y descongestione las filas de espera de esta forma le 
permitirá agilizar la entrega del servicio, el 30,7\% (117 clientes) le es indiferente que se habilite un canal adicional. Por otro lado el 10,5 \% (40 clientes) correspondientes al banco Popular es están de acuerdo que en horas pico se instale un nuevo servidor

\begin{tabular}{|c|c|c|c|c|}
\hline & & \multicolumn{2}{|c|}{$\begin{array}{l}\text { Pregunta . } 15 \text { Considera que } \\
\text { el personal que labora en la } \\
\text { entidad financiera es } \\
\text { suficiente para suplir el } \\
\text { servicio }\end{array}$} & \multirow[t]{2}{*}{ Total } \\
\hline & & No & Si & \\
\hline \multirow{11}{*}{$\begin{array}{l}\text { Pregunta : } 1 \\
\text { De que } \\
\text { entidad } \\
\text { financiera es } \\
\text { cliente }\end{array}$} & Bancolombia & 17 & 14 & 31 \\
\hline & \begin{tabular}{|l|} 
Banco de \\
Bogotá
\end{tabular} & 40 & 22 & 62 \\
\hline & BBVA & 31 & 8 & 39 \\
\hline & Bancomeva & 19 & 5 & 24 \\
\hline & \begin{tabular}{|l|} 
Banco \\
Popular \\
\end{tabular} & 29 & 13 & 42 \\
\hline & Davivienda & 10 & 22 & 32 \\
\hline & \begin{tabular}{|l|} 
Banco \\
Agrario \\
\end{tabular} & 26 & 6 & 32 \\
\hline & Bancamia & 20 & 7 & 27 \\
\hline & $\begin{array}{l}\text { Coomultrasa } \\
\text { n }\end{array}$ & 33 & 8 & 41 \\
\hline & $\begin{array}{l}\text { Banco de la } \\
\text { Mujer }\end{array}$ & 26 & 16 & 42 \\
\hline & Crezcamos & 9 & 0 & 9 \\
\hline \multicolumn{2}{|l|}{ TOTAL } & 260 & 121 & 381 \\
\hline
\end{tabular}

En lo anterior se destaca que un $68.2 \%$ (260 clientes) de los clientes consideran que el personal de las entidades financieras no es suficiente para la prestación del servicio de cada una de ellas. Por otro lado, el 31.8\% (121 clientes) de los clientes consideran que con el personal de las entidades financieras es suficiente para prestar el servicio. A si mismo 10,5\% (40 clientes) de los clientes de banco Bogotá consideran que el personal que labora en las entidades es insuficiente con relación a la cantidad de servicios y clientes de la entidad.

Por lo anterior se concluye que es necesario el aumento del personal para que contribuya a mejorar el servicio en cuanto a tiempos de respuesta, agilidad y calidad para la satisfacción del cliente, ya que en la tabla tres se identificó que los clientes deben esperar más de 8 minutos en la fila para ser atendidos y en la tabla cuatro los clientes están de acuerdo con que se habilite un canal adicional en las horas pico.

\section{ANALSIS DE CORRELACIONES DE LOS CLIENTES DE LAS ENTIDADES FINANCIERAS DEL MUNICIPIO DE PAMPLONA}

En la siguiente tabla de correlaciones se identificarán las correlaciones existentes entre las preguntas aplicadas a los clientes de las entidades financieras del municipio de pamplona, a su vez se analizó la regresión y se determina la inferencia y su respectiva ANOVA (análisis de varianza) 
Resumen de modelo y estimaciones de parámetro

\begin{tabular}{|c|c|c|c|c|c|c|c|c|c|}
\hline \multicolumn{10}{|c|}{$\begin{array}{l}\text { Variable dependiente: Pregunta : } 13 \text { Califique el nivel de importancia que le otorga los } \\
\text { funcionarios antes sus solicitudes }\end{array}$} \\
\hline \multirow{2}{*}{ Ecuación } & \multicolumn{5}{|c|}{ Resumen del modelo } & \multicolumn{4}{|c|}{ Estimaciones de parámetro } \\
\hline & R cuadrado & $\mathrm{F}$ & gl1 & $\mathrm{gl} 2$ & Sig. & Constante & b1 & b2 & b3 \\
\hline Lineal & 0,513 & 399,652 & 1 & 379 & 0 & 1,123 & 0,614 & & \\
\hline Logaritmico & 0,466 & 330,203 & 1 & 379 & 0 & 1,4 & 1,447 & & \\
\hline Inverso & 0,366 & 218,55 & 1 & 379 & 0 & 3,881 & $-2,71$ & & \\
\hline Cuadrático & 0,521 & 205,527 & 2 & 378 & 0 & 1,687 & 0,171 & 0,08 & \\
\hline Cúbico & 0,522 & 137,017 & 3 & 377 & 0 & 2,071 & $-0,33$ & 0,28 & $-0,02$ \\
\hline Compuesto & 0,443 & 301,019 & 1 & 379 & 0 & 1,457 & 1,258 & & \\
\hline Potencia & 0,414 & 267,468 & 1 & 379 & 0 & 1,603 & 0,549 & & \\
\hline $\mathrm{s}$ & 0,333 & 189,235 & 1 & 379 & 0 & 1,418 & $-1,04$ & & \\
\hline Crecimiento & 0,443 & 301,019 & 1 & 379 & 0 & 0,376 & 0,23 & & \\
\hline Exponencial & 0,443 & 301,019 & 1 & 379 & 0 & 1,457 & 0,23 & & \\
\hline
\end{tabular}

El cuadro anterior se identifican las regresiones Lineal, Logarítmico, Inverso, Cuadrático, Cúbico, Compuesto, Potencia, S, Crecimiento y Exponencial, se observa que la mayor correlación de Pearson la presenta la regresión cubica con valor de 0.522 y con $\mathrm{R}=0,722$, siendo esta correlación regular con una tendencia a ser aceptable, por otro lado la correlación lineal está determinada por un valor de 0,513 , con un $\mathrm{R}$ equivalente de 0,716 que coincide con la correlación del cuadro anterior, con respecto a los parámetros de la estimación de la regresión lineal cuyo modelo está definido por $\overline{\mathrm{y}}=$ $\mathrm{bx}+\mathrm{c}$ y su tendencia o pronostico está definido como $\bar{y}=0,614 \mathrm{x}+1,123$

\begin{tabular}{|c|r|r|r|}
\hline \multicolumn{4}{|c|}{ Resumen del modelo } \\
\hline$R$ & R cuadrado & $\begin{array}{c}\text { R cuadrado } \\
\text { ajustado }\end{array}$ & $\begin{array}{c}\text { Error } \\
\text { estándar de } \\
\text { la }\end{array}$ \\
\hline 0,72 & 0,513 & 0,512 & 0,487 \\
\hline
\end{tabular}

En la siguiente tabla de ANOVA se puede identificar la variabilidad de los datos del objeto de estudio

\begin{tabular}{|c|c|c|c|c|c|}
\hline \multicolumn{6}{|c|}{ ANOVA: } \\
\hline$\alpha$ & Suma-de-cuadrados & Glos & Media-cuadráticas & $\mathrm{Fss}$ & Sig.w \\
\hline Regresióna: & 94,855 & 10 & 94,855 & 399,652 &, 0000 \\
\hline Residuos & 89,953 & 379 & ,237 & & \\
\hline Totala & 184,808 & 38000 & & & \\
\hline
\end{tabular}

El análisis de la ANOVA (análisis de varianza) se identificó que la hipótesis (significancia) nos dio un valor de, $000 \mathrm{y}$ el entorno (hallado de varios experimentos preestablecido estadísticamente y comparados con el del estudio), y con un F observada 1 y 379 grados de libertad y con un nivel de confianza del $95 \%$ y comparado con los puntos porcentuales de la distribución estándar de $\mathrm{F}$ calculado dio un valor de F observada 1 y 379 grados de libertad, un guarismo o valor de 3.90 Con el dato comparado de la tabla de Fisher identificamos que se presentan diferencias significativas entre las medias de la población de estudio, lo anterior debido a que existen mucha dispersión, variabilidad y varianza entre los datos. 
Por lo anterior se comprueba que se hace necesario mejorar la calidad del servicio y diseñar e implementar nuevos canales de servicios o cajeros que permitan brindar un mejor servicio que haga a las entidades más competitivas y de esta forma aumentar el nicho del mercado.

\section{CONCLUSIONES Y RECOMENDACIONES}

Con relación al resultado obtenido en el análisis de correlación de los empleados de las entidades financieras se identificó que se presentan diferencias significativas en los datos en la parte experimental que aplico toda vez que el $\mathrm{F}$ calculado debe ser $\leq$ al observado, a su vez el grado de significancia se identificó que ninguno de los valores de las regresiones Lineal arrojan un valor $\leq \mathrm{a}$ 0,5 con lo que se concluye que existen diferencia significativas en la fase experimental del proyecto lo que indica se deben recomendar estrategias de mejora que permitan aumentar la satisfacción de los clientes de las entidades financieras.

En el análisis descriptivo de los empleados de las entidades financieras se identificó que los tiempos que un servidor utiliza para atender a un cliente están en un intervalo de 6 a 8 minutos y entre 4 y 6 minutos, lo que quiere decir y con relación a la cantidad de clientes que manejan las entidades financieras del municipio de Pamplona es muy alto por lo que se recomienda a las entidades replantear la cantidad de servidores o puestos de servicio que permita disminuir los tiempos de atención por parte de los funcionarios de las entidades financieras y así agilizar los procesos que satisfagan las necesidades de los clientes.

Por otro lado se identificó que el tiempo que un cliente debe esperar en cola para ser atendidos por un funcionario está comprendido entre 6 y 8 y mayor 8 minutos lo que corresponde a un tiempo muy alto con relación al flujo de clientes que manejan las entidades financieras. Lo anterior da para realizar un estudio de teoría de colas, esta teoría de colas o líneas de espera están relacionados con los procesos caracterizados por llegadas aleatorias o intervalos, uno de los procesos relacionados directamente con esta herramienta son aquellas organizaciones cuya finalidad es servicio al cliente y el servicio es aleatorio Barón Bierman ,Bonini, Hausman y Warren (2000); para Aguilar, Cruz y Regalado (2014) teoría de colas o líneas de espera son aquellos clientes que esperan ser atendidos y no se incluyen al cliente que ya está siendo atendiendo, por lo anterior se recomienda realizar este estudio ya que permite determinar las falencias en cuanto a demoras y flujo del movimiento en cola y conlleve a determinar la instalación de uno o más de un nuevo canal de servicios teniendo como referente que el $61.4 \%$ de los clientes respondieron que en horas picos se instale o abran un nuevo servidor se descongestione la cola de espera que supla las necesidades y requisitos de los clientes.

Referencias Bibliografícas

Aguilar G.M.; Cruz M.A y Regalado H.F (2014). Modelo de la teoría de colas para optimizar los tiempos de espera de los pacientes de medicina general de la unidad comunitaria de salud familiar ZACAMIL, Municipio de Mejicanos, Departamento de San Salvador. Universidad del Salvador 2014.

Avila de Barón, Cecilia; Bierman, Harold, Jr; Bonini, Charles E; Hausman, Warren H (2000), Análisis cuantitativo para los negocios novena edición McGraw-Hill Interamericana 2000.

Baptista M.V y León M de F 2013, Estrategias de lealtad de clientes en la banca universal. El Sevier, Doyma. Departamento de Ciencias Administrativas, Universidad de Los Andes, Mérida, Venezuela. 
Bustamante J.C(2015), Uso de variables mediadoras y moderadoras en la explicación de la lealtad del consumidor en ambientes de servicios, coordinador del área de Marketing, Departamento de Ciencias Administrativas y Contables, Universidad de Los Andes-Táchira, Venezuela.

Chao, P. (2008). Exploring the nature of the relationships between service quality and customer loyalty: an attribute-level analysis. The service industries jour- nal 28 (1): 95-115

Goode y Moutinho (1996), La satisfacción del cliente Bancario en Canadá: Diferencias entre canadienses e inmigrantes de la América Latina - Revisión y traducción 2009

GURU (2003), Calidad del servicio de salud:una revisión a la literatura desde la perspectiva del marketing.

Hernández Garcia Jessika Andrea ., Ariza Garcia Jherson, Cano Calle Herminsul de Jesús ,Contreras Pineda Jorge .2013.Estandarización de una técnica para la certificación de jardines clonales de plantas de cacao(Theobroma cacao ) usando marcadores moleculares rapd. Bistua:Revista de la Facultad de Ciencias Básicas.10(2):75-84

Iguarán, R., Chávez, E. y Pérez, J. (2006). Satisfacción de los clientes del servicio médico odontológico de la universidad del Zulia. Revista Venezolana de Ciencias Sociales UNERMB, Vol. 10, No 1, pp. 176-203. International Standardization Organization (ISO).

Ko, Y. y Pastore, D. (2005). A hierarchical model of service quality for the recreational sport industry.Sport Marketing Quarterly, Vol. 14, pp. 84-97.

Newstrom, John W. (2007). Comportamiento humano en el trabajo. (12a Ed.). México: Mc

Graw Hill-Interamericana.

Peralta (2004) Rol de las expectativas en el juicio de satisfacción y calidad percibida del servicio Universidad Tarapacá Arica chile

Pizzo, M. (2013). Construyendo una definición de Calidad en el Servicio. Recuperado el 29 de agosto de 2013, de la fuente: http://comoservirconexcelencia. com/blog/construyendo-una-definicion-de-calidad-en-el-servicio/.html Publicaciones Vértice S.L. (2010). Atención al cliente. Editorial Vértice. España. p. p. 10. Publicaciones Vértice S.L. (2013). La calidad en el servicio al cliente. Editorial Vértice. España. p. p. 1, 3.

Romero, G. y Romero, J. (2006). Satisfacción de clientes externos de las empresas aseguradoras en el municipio Maracaibo. Revista de Ciencias Sociales, Maracaibo, Vol. 12, № 3, pp. 10. [Documento en línea]. Disponible en: http:// www.scielo.org.ve/scielo.php?pid=S1315-95182006000300011\&script=sci_arttext.

Ruyteret AI, (1997); Ghobadian et al, (1994). "Calidad del Servicio y Fidelidad del Cliente en Franquicias y Negocios Familiares en Aguascalientes" Departamento de Ciencias Económico Administrativas, Instituto Tecnológico de Aguascalientes 2013.

Sampieri Hernandez, R.y Collado Fernandez, C.(2010) Metodología de la investigación. Cuarta Edición. Editorial McGraw Hill.

Satisfacción laboral=productividad (2011), Revista Articuz. Edición de la página de internethttp://www.articulosinformativos.com.mx/Satisfaccion_en_el Trabajo_Productividad

SETO (2004), Estrategias de Lealtad de Clientes en la Banca Universal. María Virginia Baptistaa y María de Fátima León, Departamento de Ciencias Administrativas, Universidad de Los Andes, Mérida, Venezuelab Profesora, Centro de Investigaciones y Desarrollo Empresarial, Universidad de Los Andres, Mérida, Venezuela

Wang Y., Lo, H. y Hui, H. V. (2003), “The Antecedents of Service Quality and Product Quality and their Influences on Bank Reputation: Evidence from Banking Industry in China”. En: Managing Service Quality, 13: 72-83.

Zeithaml, V. y Bitner, M. (2002). Marketing de servicios. México: McGraw-Hill/Interamericana.

Martínez Bencardino, Ciro, Estadística y muestreo / Ciro Martínez Bencardino. -- 13a. ed. -Bogotá : Ecoe Ediciones, 2012. 900 p. - (Ciencias exactas. Matemáticas) 
*Para citar este artículo: Peñaranda Ayala Z.V .;Quijano Parra A .;Thomas Manzano S.. Evaluating the customer's satisfaction level the financial entities of the municipality of Pamplona.. Revista Bistua. 2019 17(1):94-111

+ Autor para el envió de correspondencia y la solicitud de las separatas: Peñaranda Ayala Z.V Facultad de Ingenierias y Arquitectura.Ingeniera Industrial. Universidad de Pamplona. /zoraima2009@hotmail.com

Recibido: Marzo15 de 2018

Aceptado: Agosto 10 de 2018 